\title{
On the accretion onto the white dwarfs in symbiotic binaries: A test with the Thomson scattering process
}

\author{
Matej Sekeráš and Augustin Skopal \\ Astronomical Institute, Slovak Academy of Sciences, \\ 05960 Tatranská Lomnica, Slovakia \\ email: msekeras@ta3.sk, email: skopal@ta3.sk
}

\begin{abstract}
We tested the origin of the nebular radiation in the symbiotic star AG Dra, during different levels of its activity. We modeled the broad wings of the OVI 1032, $1038 \AA$ resonance lines and HeII $1640 \AA$ line by the Thomson scattering process, and determined the electronscattering optical depth, $\tau_{e}$, of the symbiotic nebula. The increase of $\tau_{e}$ during active phases results from an increase in the accretion rate onto the white dwarf.
\end{abstract}

Keywords. Binaries: symbiotic, scattering, line: profiles, accretion

\section{Introduction}

Symbiotic stars (SS) are long-period interacting binaries consisting of a (G-)K-M giant star and accreting compact star, most probably a white dwarf (WD). The material, which is accreted from the giant's wind onto the WD surface, increases the temperature and luminosity of the WD to $T_{h} \approx 10^{5} \mathrm{~K}$ and $L_{h} \approx 10^{2}-10^{4} L_{\odot}$ respectively. In SS we distinguish (i) a quiescent phase, when the binary system release its energy with constant rate and temperature and (ii) an active phase when the brightness of the hot star increases by about 2-3 magnitudes in the optical and the energy in the spectrum is redistributed.The so-called symbiotic nebula represents the ionized fraction of the stellar winds from binary components by high-energy photons from the WD. It is appropriate for studying effects of Thomson scattering in a form of very shallow and wide wings of the strongest emission lines. We used the emission lines of the OVI 1032, $1038 \AA$ doublet and HeII $1640 \AA$ line observed in the far-UV spectra of AG Dra. We modeled the observed profiles in the same way as introduced by Sekeráš \& Skopal (2012).

\section{Results}

During the quiescent phases, we determined the range of $\tau_{e}=0.044-0.078$. It is assumed that during these phases the symbiotic nebula arises from ionizing a portion of the neutral wind from the giant only (the wind from the hot star is neglected). According to the model of Seaquist et al. 1984, the symbiotic nebula is spread asymmetrically around the hot star in the binary. This implies that $\tau_{e}$, which depends on the concentration of free electrons on the line of sight, will be a function of the orbital phase $\varphi$ of the binary. We investigated the theoretical function $\tau_{e}(\varphi)$ in the simplest stationary case for AG Dra. We find out that the function $\tau_{e}(\varphi)$ is significantly below the values derived from observations during quiescent phases (the mid panel of Fig.1). This implies that there must be more free electrons on the line of sight than it is given by only a typical mass-loss rate from the giant in SS, $\dot{M}_{g}=3.2 \times 10^{-7} \mathrm{M}_{\odot} \mathrm{yr}^{-1}$. In a more realistic case, the density distribution in the circumstellar environment is determined mainly by the rotation of 

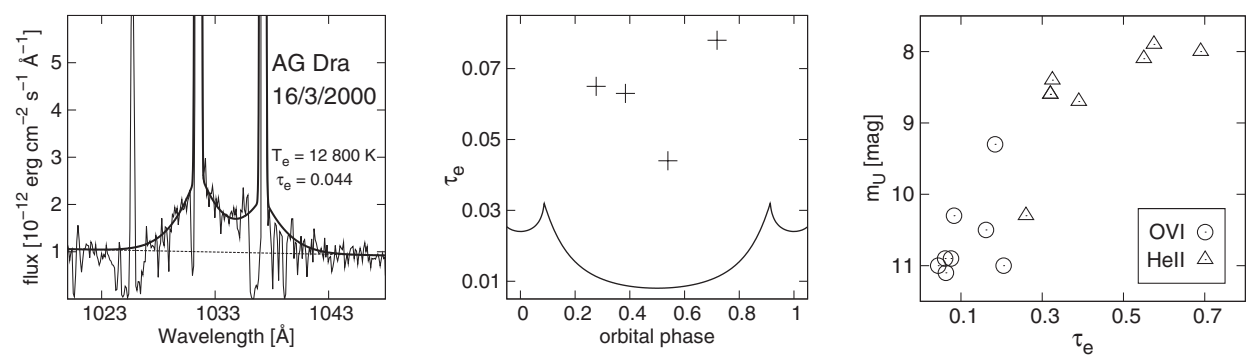

Figure 1. Left: the model of the wings of Thomson scattered OVI dublet (thick line). In the middle: the solid line represents our theoretical function of $\tau_{e}(\varphi)$ for the quiescent phase of AG Dra and the crosses are $\tau_{e}$ values derived by modeling the wings of OVI lines. Right: the relationship between $\tau_{e}$ and the star's brightness in the U filter for AG Dra.

the binary and accretion by the hot star producing regions with highly increased density (e.g., Bisikalo et al. 1995).

In the active phase we determined the range of $\tau_{e}=0.084-1.17$. The growth of the accretion rate onto the WD is supposed to change the symbiotic system from quiescent to active state, when the hot star increases its temperature and luminosity. The hot star enhances significantly the mass-loss rate in a form of the stellar wind and consequently the emission measure of the nebula (e.g., Fernández-Castro et al. 1995). The stellar wind particles are ionized by the luminous WD and thus represents a significant supplement of free electrons into the nebula. This is supported by the higher value of $\tau_{e}$. The ionized stellar wind from the white dwarf becomes a main contributor to the nebular radiation and dominates also the spectral region of the photometric $U$ filter. The activity level of the $\mathrm{SS}$ is therefore mapped with the $\mathrm{U}$ light curve, which explains the observed relation between $\tau_{e}$ and the $\mathrm{U}$ magnitude (Fig. 1 right).

\section{Conclusion}

Significant changes in $\tau_{e}$ reflect different properties of the symbiotic nebula during different levels of activity. During quiescent phases, the scattering off the free electrons from/around the accretion disk and the ionized wind from both the giant and the WD, are responsible for the observed faint OVI line wings. During active phases, $\tau_{e}$ increases by a factor of $\gtrsim 10$. This suggests an increase in the accretion rate onto the WD and consequently an increase in the mass-loss rate. The relationship between $\tau_{e}$ and the level of the activity supports this view.

\section{Acknowledgment}

The research was supported by a grant of the Slovak Academy of Sciences No. 2/0038/ 10.

M.S. acknowledges the support from the IAU.

\section{References}

Bisikalo, D. V., Boyarchuk, A. A., Kuznetsov, O. A., Popov, Y. P., \& Chechetkin, V. M. 1995, Astron. Reports, 39, 325

Fernández-Castro, T., González-Riestra, R., Cassatella, A., \& Taylor, A. R., Seaquist E. R. 1995, ApJ, 442, 366

Seaquist, E. R., Taylor, A. R., \& Button, S. 1984, ApJ, 284, 202

Sekeráš, M., Skopal A. 2012, MNRAS, (in press) 